\title{
Démocratie municipale : de la théorie à la pratique
}

\author{
Sylvain Gaudreault ${ }^{1}$ \\ Cégep de Jonquière
}

Alors que les sociétés occidentales contemporaines vivent une ère que l'on dit dominée par l'information et où la mondialisation des marchés incite paradoxalement à s'intéresser aux questions locales, il est étonnant de constater que le taux de participation aux élections municipales reste peu élevé. Plusieurs acteurs locaux et même l'Organisation de coopération et de développement économique (OCDE) ont fait état de la démobilisation des citoyens à l'égard de la politique municipale ${ }^{2}$. Par conséquent, on a la désagréable impression que le monde municipal recule au lieu d'avancer. Pourtant, l'une des principales caractéristiques de la démocratie municipale est le principe de proximité entre les contribuables et le secteur public. Cette proximité est supposée favoriser le respect des goûts et des besoins des citoyens par rapport à l'exercice des fonctions collectives.

\section{De « Petit-Bonheur » à Alma, la démocratie remise en question}

Chacun y va de ses solutions. Denis Trottier, le maire de Péribonka, au Lac-Saint-Jean, a choisi une formule choc. Habitué aux coups d'éclat, le maire Trottier a rédigé une offre d'emploi fictive qui ferait sourire si ce n'était de la tragique réalité qu'elle dénonce sur un ton ironique. Dans cette offre, la municipalité de « Petit-Bonheur» $\left(750\right.$ h.) lance un $2^{\mathrm{e}}$ concours pour le poste de maire. Les défis sont nombreux. On y trouve notamment «l'augmentation de la productivité de nos trois employés ». Le profil de la personne recherchée est rare. Le candidat devra « avoir une forte personnalité, tout en possédant une grande capacité à écouter, à analyser et à influencer, alliée à un souci pour la rigueur tout en faisant preuve de souplesse ». Parmi les exigences, on demande, outre une voiture, de «posséder une expérience diversifiée d'au moins
10 ans en gestion ». Les conditions de travail ? « Etre disponible en tout temps [...], régime de retraite non disponible [...]), rémunération de $77.03 \$$ par semaine et allocation non imposable de $38.51 \$$ par semaine $»$.

Remplacez « Petit-Bonheur » par n'importe quelle autre municipalité rurale du Québec et vous constaterez que l'offre de Denis Trottier n'est pas loin de la réalité. À ce compte-là, faut-il s'étonner que SaintStanislas, petite municipalité au nord du Lac-SaintJean, n'ait trouvé personne pour se sacrifier à la mairie lors des élections du 2 novembre 2003? Au Bas-Saguenay, le maire de Ferland-et-Boilleau a démissionné avec fracas, désabusé par les exigences d'une fonction ingrate. Trois sièges de conseillers sont restés vacants à Rivière-Éternité, deux à SainteHedwidge et un à Saint-Ludger-de-Milot. À défaut de lutte électorale, la plupart des postes ont été comblés par acclamation, comme cela était courant au XIX ${ }^{\mathrm{e}}$ siècle et au début $\mathrm{du} \mathrm{XX}^{\mathrm{e}}$, quand les gens étaient analphabètes, les femmes n'avaient pas le droit de vote et les moyens de communication étaient rudimentaires. La démocratie municipale, censée être plus proche des citoyens, est-elle malade ?

Même à Alma (31 000 h.), le feu jaune est allumé. Enclave de prospérité au Saguenay-Lac-Saint-Jean, on se bousculait au portillon pour avoir le privilège de gérer la croissance : quatre candidats à la mairie et un seul conseiller sur huit élu par acclamation le 2 novembre 2003. Cela fait autant d'organisations électorales sur le terrain pour soi-disant «faire sortir le vote $»$. Pourtant, seulement $56 \%$ des électeurs ont jugé utile de se déplacer ${ }^{3}$.

Devant ces faits, la véritable question à débattre est la suivante : quelle notion de démocratie doit s'appli- 
quer au niveau municipal ? En d'autres termes, comment améliorer la démocratie dans les villes ? Si une meilleure vie démocratique passe par l'implication des citoyens dans les structures de pouvoir, il faut d'abord se demander, en amont, quels outils nous voulons donner aux municipalités pour que ces dernières puissent remplir leurs responsabilités grandissantes.

\section{Les réformes municipales et la démocratie}

Afin de mettre en contexte les nouveaux enjeux et défis pour la démocratie municipale au Québec, il est utile de se rappeler les objectifs principaux des récentes réformes vécues dans le monde municipal.

Pour ce qui est de la réforme Harel (1999-2002) sur la réorganisation municipale, les trois principaux objectifs étaient les suivants : économies d'échelle, amélioration des services et compétitivité entre les municipalités ${ }^{4}$. La réforme Harel a eu comme principal impact la création d'un réseau de neuf villes majeures de plus de 100000 personnes sur le territoire québécois : Montréal, Québec, Longueuil, Laval, Gatineau, Saguenay, Sherbrooke, Trois-Rivières et Lévis. Ces villes regroupent $53 \%$ de la population du Québec et occupent une place stratégique importante sur le territoire. Cependant, le principal problème de ce réseau de neuf villes est son manque de préparation pour occuper la place significative qui lui revient dans le panorama politique du Québec et du Canada, tandis qu'ailleurs au pays, les grandes villes sont en train de s'organiser pour réclamer leur place dans l'échiquier constitutionnel.

Quant à la réforme Fournier (2003-2004), sous le couvert d'un retour éventuel aux municipalités d'avant les fusions, ses objectifs sont davantage l'adhésion aux nouvelles structures, l'efficacité et l'équité fiscale.

Les nouvelles structures découlant des réformes des dernières années se distinguent par leur grande complexité et leur multiplicité. À Montréal et à Québec, par exemple, les fusions puis les éventuelles « défusions » ou démembrements provoquent la création d'institutions (municipalités ou arrondissements) inégales entre elles sur le plan de la représentativité des élus, des compétences qui leur sont dévolues et de la fiscalité. Ajoutons à cela la création des Conférences régionales des élus (CRÉ) et les citoyens se perdent dans le dédale des nouvelles instances. Cette situation tend à éloigner davantage les citoyens de la vie politique à l'échelon local et les encourage à ne pas aller voter.

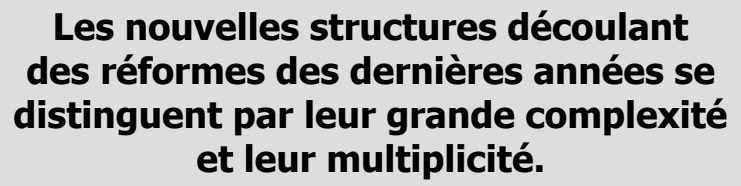

En revanche, soulignons que les prochaines élections municipales du 6 novembre 2005 se tiendront pour la première fois simultanément dans toutes les municipalités du Québec. Il reste à voir si cette manière de faire aura un impact favorable sur la vie démocratique car, de façon générale, le taux de participation aux élections municipales est peu élevé.

Ce rappel des objectifs des réformes permet de constater qu'aucun de ceux-ci ne touche la démocratie municipale comme telle. En dépit des nombreuses réformes, toute la question de la démocratie municipale au Québec reste donc ouverte ${ }^{5}$.

\section{En dépit des nombreuses réformes, toute la question de la démocratie municipale au Québec reste ouverte.}

\section{Une définition de la démocratie municipale}

Dans un premier temps, il n'est pas inutile de définir la démocratie au-delà de sa conception utopique, en tant qu'idéal de société. À cet effet, il est déjà plus concret de se pencher sur le processus de démocratisation en tant que tel, soit la démocratie participative. Lagacé et Prévost ${ }^{6}$ vont en ce sens en disant que « la démocratie participative est un processus qui vise, entre autres, à accroître la capacité citoyenne des gens par le biais de l'accessibilité à la formation et à l'éducation ainsi qu'aux informations pertinentes ». Plus loin, ces mêmes auteurs ${ }^{7}$ ajoutent : « La démocratie participative est un mode de gestion qui s'actualise dans la création d'une gouvernance. Cette gouvernance naît de la nécessité de répondre à un besoin commun par l'entremise d'un projet collectif ». Bien qu'intéressante parce qu'elle tient compte à la fois de l'éducation à la citoyenneté et de l'élaboration d'une 
vision, facteurs en amont de la participation des citoyens aux affaires publiques, cette définition en deux volets reste néanmoins incomplète.

Vincent Lemieux, politologue et professeur émérite de l'Université Laval, s'inspire notamment des travaux $\mathrm{du}$ politologue états-unien Elmer E. Schattschneider pour aller plus loin dans la définition de la démocratie. Il propose en effet une définition la plus pragmatique possible comportant six concepts, ceux-ci formant les conditions de la démocratie locale et régionale : «Démocratie : système politique compétitif dans lequel des leaders ou des organisations sont responsables devant la population et politisent les problèmes collectifs, en popularisant des solutions de façon à impliquer la population ».

Voyons plus en détail chacun des éléments de cette définition :

- Compétition : par ce terme, on entend les débats et les discussions entre diverses tendances ou opinions. La compétition est un outil contre les dérives du pouvoir central.

- Leaders et organisations : un ingrédient essentiel à la démocratie est la présence de quelqu'un ou d'une organisation en mesure d'exercer le leadership et de défendre une cause (ou des causes). C'est ce qu'on appelle aussi « avoir de la vision ».

- Responsabilité : les personnes ou les organisations exerçant le leadership doivent être à l'écoute de la population, c'est-à-dire répondre à ses inquiétudes, à ses critiques et à ses aspirations.

- Politisation des problèmes collectifs : prendre position et soulever les problèmes publiquement pour qu'ils soient résolus font partie des fonctions des leaders démocratiques. En d'autres termes, les leaders ont l'obligation de traiter ou d'affronter les problèmes de la collectivité.

- Popularisation des solutions : un problème étant l'écart entre un désir et la réalité, il faut de la volonté politique pour combler cet écart et, ainsi, proposer des solutions partagées par le plus grand nombre.

- Implication de la population : il doit y avoir une participation la plus large possible aux décisions structurantes (à distinguer des décisions courantes ou « de routine ») qui concernent la collectivité. Pour ce faire, les leaders et les organisations politiques doivent éviter les promesses trop précises qui font en sorte qu'une fois élus, ils se trouvent incapables de les remplir en raison des divers événements qui interviennent dans la gestion gouvernementale courante. Du coup, les citoyens perdent confiance à l'endroit des politiciens, ce qui peut expliquer la baisse de la participation populaire aux élections ${ }^{8}$.

\section{Les fondements théoriques de la démocratie municipale}

\section{La démocratie et la communauté}

La question de la démocratie municipale renvoie directement à la notion de communauté en tant qu'organisation sociétale à petite échelle. Selon Proulx ${ }^{9}$, la communauté se définit comme étant «un territoire délimité sur lequel les habitants gèrent collectivement des fonctions diverses (biens et services) dont ils ont le besoin commun. La communauté se situe qualitativement en aval de la simple collectivité, par l'identité, l'intimité, l'interaction, les complicités et la solidarité ». Les municipalités et les arrondissements du Québec correspondent en tous points à cette définition de la communauté.

La démocratie et l'esprit de communauté apparaissent indissociables. Favoriser l'un apporte le développement de l'autre. L'exercice de la démocratie dans la conduite des affaires publiques est un des prérequis essentiels au développement local cohérent et durable ${ }^{10}$. Toujours selon Proulx ${ }^{11}$, l'amélioration de l'organisation communautaire repose préalablement « sur un solide diagnostic de la situation puis sur un important effort de sensibilisation de la population de l'aire communautaire ». Autrement dit, ce n'est pas tout de constater le déficit démocratique dans les municipalités; il faut aussi proposer des procédures, des techniques et des mécanismes (parlementarisme municipal, compétition, leadership, responsabilité, information, partis politiques, etc.) pour améliorer la démocratie à l'échelle municipale et, du coup, bonifier l'organisation de la communauté.

D'autres avant nous ${ }^{12}$ ont fait largement état de la démobilisation des citoyens à l'égard de la politique 
municipale. Chacun a suggéré ses solutions, ces dernières se regroupant toutes sous le dénominateur commun d'une meilleure vie démocratique axée sur l'implication des citoyens dans les structures de pouvoir. Proulx ${ }^{13}$ abonde dans le même sens en ce qui concerne la communauté lorsqu'il affirme: «L'organisation et le développement de l'aire communautaire par ce qu'il est convenu d'appeler l'appropriation (empowerment) et la solidarité se présentent aujourd'hui comme la voie tout indiquée pour contrer les effets de la démobilisation sociale de la mondialisation $»$.

\section{L'État local se caractérise d'abord par la présence d'un sentiment d'appartenance chez les citoyens.}

\section{La démocratie et l'État local}

Plusieurs spécialistes de l'analyse spatiale axent leurs recherches sur le fonctionnement politique et administratif interne des aires sous l'angle de l'État local. Par État local, nous entendons « l'ensemble des organisations publiques (emploi, santé, postes, transport, éducation, etc.) plus ou moins décentralisées qui interviennent dans une aire ou un espace donné $\gg{ }^{14}$.

L'État local se caractérise d'abord par la présence d'un sentiment d'appartenance chez les citoyens. L'appartenance à un territoire est un facteur fondamental garantissant la solidarité communautaire. D'ailleurs, entretenir la solidarité communautaire provoque des effets bénéfiques sur les organisations et sur les forces sociales, culturelles et économiques. C'est ainsi que le sentiment d'appartenance à une communauté favorise l'efficacité économique et démocratique ${ }^{15}$.

La deuxième caractéristique de l'État local est sans aucun doute le principe de proximité entre les contribuables et le secteur public. Cette proximité - particulièrement présente dans les municipalités - favorise le respect des goûts et des besoins des citoyens par rapport à l'exercice des fonctions collectives. Selon Collin ${ }^{16}$, la proximité entre les élus et les citoyens est une caractéristique fondamentale du gouvernement municipal. Sur le terrain et dans le contexte des récentes réformes vécues au Québec, cette proximité peut se traduire par une révision du fonctionnement des rapports entre trois parties: le maire, le conseil municipal (les conseillers) et la population.

Comment ? Proulx ${ }^{17}$ fait ressortir que, dans l'État local, «les goûts et les préférences de la population s'expriment localement par un mode de représentation démocratique prenant la forme d'une multitude de petits groupes d'intérêts (organisations collectives) qui exercent des pressions et effectuent des demandes auprès des autorités locales ».

Cette façon de pratiquer la démocratie est cependant critiquée, notamment dans l'État local de Saguenay que d'aucuns perçoivent comme étant dominé par quelques groupes d'intérêts. En effet, des acteurs locaux reprochent à l'administration actuelle ses liens trop étroits avec certains «amis du pouvoir» et déplorent la coupure entre les citoyens et les lieux de décision, parlant même de « déficit démocratique $»{ }^{18}$.

Face à ce constat, quelques pistes de solutions sont identifiées :

- améliorer l'implication des citoyens dans les prises de décisions, notamment par la mise en œuvre d'une forme de parlementarisme municipal ${ }^{19}$;

- créer des partis politiques municipaux ${ }^{20}$;

- appliquer le «budget participatif» dans lequel les citoyens seraient impliqués;

- favoriser une décentralisation plus grande vers les arrondissements;

- tenir compte de l'importance des médias pour diffuser l'information et pour mettre en œuvre une " démocratie informationnelle " visant à faire connaître les enjeux à la population;

- développer la e-démocratie (Internet) comme nouvel espace de participation citoyenne ${ }^{21}$.

Encore une fois, Proulx ${ }^{22}$ n'exprime pas autre chose lorsqu'il affirme : «Le pouvoir local s'exerce plus ou moins largement ainsi par un système de relations consensuelles que la démocratie participative cherche à bonifier. La rationalité administrative s'impose dans ce contexte hétérarchique en misant sur la bonne volonté de collaboration entre les décideurs publics, laquelle est favorisée par divers mécanismes institutionnels ». 


\section{Conclusion}

La démocratie municipale est l'enfant pauvre des récentes réformes entreprises par le gouvernement du Québec. Les neuf nouvelles grandes villes issues de la réorganisation vivent présentement une étape charnière de leur courte histoire : elles doivent d'abord prendre conscience de leur place prépondérante sur l'échiquier politique québécois et canadien, pour ensuite assumer ce rôle.

En théorie, les municipalités répondent mieux aux besoins des citoyens, respectent le sentiment d'appartenance, desservent mieux les services publics, sont moins bureaucratiques et favorisent une plus grande participation de la population. Toutefois, la pratique démontre que les élus locaux se responsabilisent difficilement parce qu'ils manquent d'expertise, leur assise fiscale est insuffisante et leurs pouvoirs sont limités.

Les six critères élaborés par le professeur Lemieux pour définir la démocratie peuvent éclairer les acteurs locaux. Chacune de ces six conditions est nécessaire. Lorsqu'il en manque une, la démocratie est alors boiteuse. À la lumière de cette définition, on peut affirmer que, globalement, le constat actuel est peu reluisant en matière de démocratie municipale. Toutefois, l'espoir est permis pour les vingt prochaines années alors qu'il semble se dégager un consensus dans les divers milieux locaux à l'effet de favoriser une plus grande participation des citoyens dans les lieux de pouvoir. Mais ce n'est pas tout: valoriser l'action municipale en donnant aux élus les moyens de leurs ambitions sera la bougie d'allumage d'une implication plus grande des citoyens.

\section{La démocratie municipale est l'enfant pauvre des récentes réformes entreprises par le gouvernement du Québec.}

La démocratie municipale s'inscrit dans un cadre théorique à deux volets: la communauté et l'État local. En effet, les recommandations favorables à une meilleure vie démocratique ont des effets bénéfiques sur la communauté, car elles favorisent une prise en charge par le milieu contre la démobilisation sociale. En outre, améliorer la démocratie participative à l'échelle municipale étend la participation populaire par-delà les groupes d'initiés. Pour ce faire, il n'est cependant pas inutile de mettre sur pied un certain nombre de mécanismes institutionnels dont, entre autres, les partis politiques, le parlementarisme municipal et une meilleure circulation de l'information.

\section{Notes et références}

1 Sylvain Gaudreault est professionnel au Cégep de Jonquière, chroniqueur au journal Le Quotidien et avocat.

2 Lagacé, Mélanie et Paul Prévost (2003), « Démocratie, edémocratie et gouvernance locale: réflexion sur les nouvelles voies de solution aux enjeux actuels de développement collectif », texte publié dans le cadre des Rencontres internationales « Démocratie et management local », Québec, 20-23 mai.

3 Gaudreault, Sylvain (2003), «Choisir entre sa ville et la réalité de Loft Story... », Le Quotidien, 13 novembre, p. 9.

4 Collin, Jean-Pierre (2003), « Villes d'agglomération et renforcement des instances supra-locales : enjeux et défis pour la démocratie municipale », présentation faite à l'occasion du séminaire «Démocratie et gouvernance», Vision Saguenay 2025, Université du Québec à Chicoutimi, 12 décembre.

5 Gaudreault, Sylvain (2003), «Un débat à organiser, celui de la démocratie municipale », Le Quotidien, 18 décembre, p. 9.

6 Lagacé et Prévos, op. cit., p. 5.

7 Idem, p. 12.

8 Gaudreault, Sylvain (2003), «Compte rendu du séminaire $\mathrm{n}^{\circ} 6$ de Vision Saguenay 2025 : Démocratie », essai rédigé dans le cadre de la Maîtrise en études et interventions régionales, Université du Québec à Chicoutimi, texte non publié.

9 Proulx, Marc-Urbain (2002), L'économie des territoires au Québec, Sainte-Foy, Presses de l'Université du Québec, p. 115.

10 Lagacé et Prévost, op. cit.

11 Proulx, op. cit.

12 Lagacé et Prévost, op. cit.

13 Proulx, op. cit., p. 116.

${ }^{14}$ Idem.

15 Mouvement Territoires et Développement (1999), «L'approche territoriale au Québec», revue Organisations et Territoires, vol. 8, n ${ }^{\text {os }} 1$ et 2, printemps-été 1999. 
16 Collin, op. cit.

17 Proulx, op. cit., p. 117.

18 Vision Saguenay 2025 (2003), «Compte rendu du séminaire : la démocratie », texte publié sur le site de Vision Saguenay 2025, http://www.uqac.ca/vsag2025/.
19 Collin, op. cit.

20 Côté, Daniel (2003), «La création de partis municipaux s'impose », Progrès-Dimanche, 17 août, p. A6.

21 Lagacé et Prévost, op. cit.

22 Proulx, op. cit., p. 117. 